\title{
Dynamiques régionales de la production laitière : France - Allemagne - Royaume-Uni
}

\section{Claire Mosnier et Christine Wieck}

\section{(2) OpenEdition}

Édition électronique

URL : http://journals.openedition.org/economierurale/3748

DOI : 10.4000/economierurale.3748

ISSN : 2105-2581

Éditeur

Société Française d'Économie Rurale (SFER)

\section{Édition imprimée}

Date de publication : 30 novembre 2012

Pagination : 132-146

ISSN : 0013-0559

\section{Référence électronique}

Claire Mosnier et Christine Wieck, «Dynamiques régionales de la production laitière : France -

Allemagne - Royaume-Uni », Économie rurale [En ligne], 332 | novembre-décembre 2012, mis en ligne le 30 novembre 2014, consulté le 01 mai 2019. URL : http://journals.openedition.org/ economierurale/3748; DOI : 10.4000/economierurale.3748 


\section{Dynamiques régionales de la production laitière France - Allemagne - Royaume-Uni}

Claire MOSNIER • INRA UR 1213 Herbivores, Clermont-Ferrand

Christine WIECK • University of Bonn, Institute for Food and Resource Economics, Bonn, Germany

Depuis 1992, les règles concernant le transfert des quotas laitiers varient fortement d'un pays à l'autre. Dans cette étude, les auteurs analysent la répartition de la production régionale de lait et son évolution en France, en Allemagne et au Royaume-Uni dont les systèmes de quota sont contrastés : la France (le plus interventionniste), l'Allemagne et le Royaume-Uni (le plus libéral). Leurs déterminants sont ensuite comparés. (NDLR)

\section{Introduction}

D epuis 1984 , la production laitière est régulée dans l'Union européenne (UE) par un système de quotas. Ils correspondent à des droits à produire attribués à chaque pays selon leurs références historiques. Ces références ont ensuite été réparties entre les éleveurs ou, dans le cas du Royaume-Uni, entre cinq Offices de commercialisation du lait. Depuis 1992, les États membres disposent de plus grandes marges de manœuvre pour gérer les transferts de quotas entre les exploitations et les régions. Ces aménagements " à la carte » ont résulté en des systèmes de transferts de quotas très divers selon les pays membres. Le Royaume-Uni a ainsi laissé le marché orienter l'attribution de quota alors qu'en France, leur transfert est très contrôlé par l'administration (Barthélémy et David, 1999) $)^{1}$. Ces systèmes de quota devraient être abolis en 2015. Les différents pays d'Europe se trouveront alors dans une situation proche de celle qu'a expérimentée le Royaume-Uni. Comparer les dynamiques de la production laitière du

1. Barthélémy D, David J. (1999). L'agriculture européenne et les droits à produire. INRA (éd.), $434 \mathrm{p}$.
Royaume-Uni à celle d'autres pays permettrait ainsi de se rendre compte de l'effet qu'ont eu les quotas sur l'organisation spatiale de la production et des conséquences potentielles de leur suppression.

Différentes études ont analysé les caractéristiques des systèmes laitiers (Butault et al., 1990 ; Chatellier et al., 2008) ${ }^{2}$, la répartition géographique de la production en France (Ben Arfa et al., 2009a) ${ }^{3}$ et cherché à comprendre les déterminants de leur répartition géographique (Daniel, 2003 ; Isik, $2004)^{4}$. D'autres se sont plus particulièrement intéressées aux déterminants de l'évolution structurelle des exploitations laitières

2. Butault J.-P., Cyncynatus M., Hassan D. (1990). The comparative advantages in the European agricultural. Économie Rurale, n' ${ }^{\circ}$ 197, p. 15-21. Chatellier V., Pflimlin A., Perrot C. (2008). La production laitière dans les régions de l'arc Atlantique européen. INRA, Production Animale, vol. 21, n' ${ }^{\circ}$, p. 427-440. 3. Ben Arfa N., Rodriguez C., Daniel K. (2009a). Dynamiques spatiales de la production agricole en France. Revue d'économie régionale et urbaine, $n^{\circ} 4$, p. 807-834.

4. Daniel K. (2003). Concentration et spécialisation : quel schéma pour l'agriculture communautaire ? Économie et Prévision, vol. 158, n ${ }^{\circ}$ 2, p. 105-120. - Isik M. (2004). Environmental regulation and the spatial structure of the US dairy sector. American Journal of Agriculture Economics, vol. 86, $\mathrm{n}^{\circ} 4$, p. 949-952. 
en France (Ben Arfa et al., 2009b ; Dervillé et al., 2011) $)^{5}$ et en Europe (Zimmermann et Heckelei, 2010) ${ }^{6}$.

Cependant, il n'existe pas à notre connaissance d'analyse des déterminants - techniques, économiques (dont les coûts de production du lait), et sociaux - de la répartition de la production de lait entre les régions selon les pays européens.

Dans cette étude ${ }^{7}$, nous analysons la répartition géographique et les dynamiques de la production laitière régionale des trois premiers producteurs de lait en Europe : le Royaume Uni, la France et l'Allemagne. Nous comparons leurs déterminants. Ces pays ont des politiques contrastées en matière de transferts interrégionaux des quotas laitiers.

- La France est le pays le plus interventionniste des trois. Avant 2011, les quotas étaient gérés très localement, au niveau des départements (96 départements métropolitains d'une superficie moyenne de $6000 \mathrm{~km}^{2}$ ) et contrôlés par l'administration. Le transfert de quota ne peut se faire indépendamment de la terre. Depuis, ce maillage a été réduit à neuf bassins laitiers.

- Le Royaume-Uni a au contraire opté depuis 1993 (Alliance Environnement,

5. Ben Arfa N., Rodriguez C., Daniel K., Shonkwiler J.-S. (2009b). Spatial structure of the French dairy sector: a spatial HAC estimation. 3rd World Conference of the Spatial Econometrics Association, July 8-10, Spain. http://www.ub.es/sea2009.com/ Papers/76.pdf

Dervillé M., Cahuzac E., Raboisson D., Maigne E., Allaire G. (2011). Déterminants individuels et territoriaux de la restructuration laitière. Renc. Rech. Ruminants, $\mathrm{n}^{\circ} 18$, p. 373-376.

6. Zimmermann A., Heckelei T. (2010). Structural change of European dairy farm- a cross regional analysis. 114th Seminar of the European Association of Agricultural Economists, April 15 - 16, Berlin, Germany.

7. Ce travail a été réalisé lorsque l'auteur était en séjour postdoctoral à l'université de Bonn. Il s'intègre à un projet ayant pour but d'estimer des fonctions de coûts de production.
2008) ${ }^{8}$ pour un marché des quotas sans lien à la terre et sans entrave géographique (excepté les îles écossaises) soit une aire de $240000 \mathrm{~km}^{2}$ environ.

- L'Allemagne est dans une situation intermédiaire avec des régions d'échange de quotas qui correspondent aux Länder qui font à peu près le double de superficie des départements français. Les transferts de quota ne sont plus liés à la terre depuis 1993 et, depuis 2007, ces zones d'échange ont été réduites à deux : l'ex-Allemagne de l'Ouest et l'Ex-Allemagne de l'Est.

Notre hypothèse est que plus le pays est libéral en matière de transfert de quotas, plus les mouvements interrégionaux sont importants et en faveur des régions qui présentent des avantages comparatifs. Les données régionales, sur la période 1996-2008, sont issues d'Eurostat et du RICA. Les analyses font appel à des indicateurs géographiques pour caractériser la distribution spatiale et son évolution et à des coefficients de corrélation pour mettre en évidence les relations entre production laitière et caractéristiques régionales.

\section{Matériel et méthodes}

\section{Indicateurs retenus et hypothèses}

Nous analysons la densité de production laitière de chaque région. Elle prend en compte la taille du cheptel et les rendements laitiers et permet de comparer des régions de superficie différente. Cette densité est exprimée par $\mathrm{km}^{2}$ plutôt que par hectare de Surface agricole utile (SAU) afin de prendre en compte la proximité et la concentration géographique de la production sur le territoire (y compris les espaces non agricoles). Nous supposons que c'est à ce niveau que les économies externes et les

\footnotetext{
8. Alliance Environnement (2008). Evaluation of the environmental impact of milk quotas. Report for DG Environment, European Commission. http://ec.europa.eu/agriculture/eval/reports/milk_quo t_ei/shortsum_en.pdf
} 
nuisances sont le mieux appréciés (coût de collecte, concentration des pollutions sur un territoire, etc.).

Les déterminants potentiels de l'organisation spatiale de la production et de sa dynamique sont supposés en lien avec la théorie des avantages comparatifs (Mosnier et Wieck, 2010) ${ }^{9}$. Cette théorie explique qu'une région aura intérêt à produire du lait si le gain obtenu par l'allocation des ressources à la production laitière plutôt qu'à d'autres activités est plus élevé que celui obtenu par l'autre région. Cela signifie qu'il faut à la fois considérer les performances économiques de l'activité d'élevage mais aussi celles des activités alternatives. Les performances de l'activité de production laitière dépendent $a$ priori des facteurs de production disponibles et de leur coût, de la technologie employée et de son efficacité.

Nous supposons que la marge nette des exploitations laitières a un effet positif sur la production régionale de lait (elle incite les éleveurs à produire plus), et les coûts de production, un effet négatif (la compétitivité de la production régionale sera meilleure). Un prix du lait payé aux éleveurs faible pourra être plus favorable au développement de la filière aval bien qu'il puisse démotiver les éleveurs. Des technologies de production de lait plus intensives par animal (rendement laitier) et par surface (chargement) sont parfois associées à de plus faibles coûts de production (Wieck et Heckelei, 2007) ${ }^{10}$. Les exploitations de grande taille bénéficient aussi potentiellement d'économies d'échelle qui permettent de réduire les coûts de production unitaires

9. Mosnier C., Wieck C. (2010). Determinants of spatial dynamics of dairy production: a review. http://www.ilr1.uni-bonn.de/agpo/publ/dispap/ download/dispap10_02.pdf

10. Wieck C., Heckelei T. (2007). Determinants, differentiation, and development of short-term marginal costs in dairy production: an empirical analysis for selected regions of the EU. Agricultural Economics, vol. 36, n 2 , p. 203-220.
(Mosheim et Lovell, 2009) ${ }^{11}$ et favorisent l'augmentation de la production régionale (Isik, op. cit.) La structure spatiale de la production peut influer sur les coûts des facteurs et des produits et sur l'efficacité de la production. Elle affecte en effet les coûts de transport (Beckmann et Thisse, 2000) ${ }^{12}$, notamment les coûts de collecte du lait (Daniel, op. cit.) L'agglomération d'élevages laitiers créerait des économies externes en attirant des industries et services spécialisées (alimentation, machines agricoles, vétérinaires...). Elle favoriserait par ailleurs la restructuration de la filière laitière en très grosses unités. À l'opposé, elle serait source d'externalités négatives qui ont principalement trait aux nuisances et aux pollutions : plus les élevages sont nombreux et plus leurs impacts environnementaux cumulés sont importants. Il est ainsi probable que la production laitière se développe plus là où elle est déjà fortement implantée ou à proximité de régions très productrices.

Nous supposons aussi que les terres avec des potentiels élevés (rendements céréaliers forts, faible part de surfaces classées « défavorisées ») sont plus favorables au développement des cultures que de l'élevage. Une part importante d'agriculteurs «jeunes » et un territoire dans lequel les activités agricoles occupent une place importante (mesurées ici par la part des surfaces agricoles et la part d'emploi agricole) reflètent $a$ priori une région où le secteur agricole est important, ce qui serait plutôt favorable à une augmentation de la production laitière. De plus, un niveau de vie peu élevé (estimée par le PIB/hab., taux de chômage) et une faible densité de population laisse

11. Mosheim R., Lovell. CAK. (2009). Scale economies and inefficiency of U.S dairy farms. American Journal of Agricultural Economics, vol. 91, $\mathrm{n}^{\circ} 3$, p. 777-794.

12. Beckmann M.J., Thisse J.-F. (2000). The location of production activities. In Nijkamp P. (ed.), "Handbook of Regional and Urban Economics", p. 21-95. 
Tableau 1. Les indicateurs retenus : source et relation attendue avec l'accroissement des densités de production régionale

\begin{tabular}{l|c|l|c}
\hline \multicolumn{1}{c|}{ Indicateur } & Source & \multicolumn{1}{c}{ Calcul } & $\begin{array}{c}\text { Effet } \\
\text { attendu }\end{array}$ \\
\hline Densité production lait & EUROSTAT & $\begin{array}{l}\text { Lait produit (agr_r_milkpr) / surface } \\
\text { régionale en km² }\end{array}$ & + \\
\hline Marge nette du lait & RICA & voir *note ci-dessous & + \\
\hline Coûts spécifiques du lait & RICA & voir *note ci-dessous & - \\
\hline Prix du lait & RICA & $\begin{array}{l}\text { recette de la vente de lait / } \\
\text { production lait }\end{array}$ & - \\
\hline Rendement laitier, chargement & RICA & Variable non modifiée & + \\
\hline Production lait / ferme & EUROSTAT & $\begin{array}{l}\text { Production de lait total (agr_r_milkpr) / } \\
\text { nombre de ferme ayant des bovins } \\
\text { laitiers >2 ans (ef_r_nuts_100) }\end{array}$ & + \\
\hline Rendement des céréales & EUROSTAT & Variable non modifiée (agr_r_crops) & - \\
\hline Zone défavorisée & EUROSTAT & $\begin{array}{l}\text { Superficie des exploitations en zones } \\
\text { défavorisées (ef_r_nuts_005) / surface } \\
\text { régionale en km² }\end{array}$ & + \\
\hline Part des exploitants $<35$ ans & EUROSTAT & $\begin{array}{l}\text { Nb Exploitants (UTA) <35 ans } \\
\text { (ef_r_nuts_119) / Nb Exploitants (UTA) } \\
\text { total (ef_r_nuts_117) }\end{array}$ & + \\
\hline Part des surfaces agricoles & EUROSTAT & $\begin{array}{l}\text { Surface agricole (ef_r_nuts_002) / } \\
\text { surface régionale en km² }\end{array}$ & + \\
\hline Taux d'emploi en agriculture & EUROSTAT & Variable non modifiée & + \\
\hline Taux de chômage & EUROSTAT & Nb de chômeurs/ population totale & + \\
\hline PIB /hab. & EUROSTAT & Variable non modifiée & - \\
\hline Densité de population & EUROSTAT & $\begin{array}{l}\text { Population régionale (demo_r_d2jan) } \\
\text { surface régionale en km² }\end{array}$ & - \\
\hline
\end{tabular}

* Note : pour calculer les ratios économiques à partir du RICA, nous avons suivi la méthodologie définie dans le rapport de la Commission européenne (2007) sur les exploitations spécialisées en production laitière. Les coûts variables incluent les charges associées aux surfaces fourragères et aux surfaces destinées à l'alimentation animale, à l'achat d'aliments (grossier et concentré) et aux divers coûts spécifiques à l'élevage d'herbivore (dont les frais vétérinaires). Ces coûts sont affectés au prorata de la part des UGB laitiers sur les UGB herbivores. Les frais généraux (entretien du matériel, énergie, salariés etc.), les amortissements et les autres intrants non spécifiques sont attribués selon la part du produit « lait et subventions associées » dans le produit total de l'exploitation. La marge nette fait le bilan entre les recettes et subventions associées à la production laitière et toutes les charges.

supposer que la région accepterait mieux les externalités négatives induites de forte densité de production. De plus, les moindres possibilités pour les éleveurs de trouver un emploi plus rémunérateur les inciteraient à rester dans l'élevage laitier.

\section{Les données}

Nous avons veillé à travailler sur des entités de taille comparable entre les pays car, $a$ priori, plus le niveau d'agrégation est élevé, moins les transferts entre régions sont importants (Alliance Environnement, op. cit. ; Ben Arfa et al., 2009a). Nous avons retenu : -21 régions administratives françaises (superficie : moy. $25000 \mathrm{~km}^{2}$ et écart type : $11000 \mathrm{~km}^{2}$ ) ;

- 13 länder allemands (superficie : moy. $27000 \mathrm{~km}^{2}$ et écart type : $16000 \mathrm{~km}^{2}$ ); - 11 régions au Royaume-Uni qui ont les contours des nations, et dans le cas de l'Angleterre des "Government Office Regions" (superficie : moy. $22000 \mathrm{~km}^{2}$ et écart type : $18000 \mathrm{~km}^{2}$ ).

Ce maillage est référencé dans les statistiques européennes par l'échelle NUTS 1 pour l'Allemagne et NUTS 2 pour la France et le Royaume-Uni. Les régions correspondant presque exclusivement à des agglo- 
mérations (Londres, Berlin, Hambourg, Brème) ont été supprimées.

Tous les indicateurs sont des données agrégées par région. Ils ont été calculés à partir de deux sources de données, Eurostat et le Réseau d'information comptable agricole (RICA). Nous avons extrait d'Eurostat les variables régionales agrégées relatives aux caractéristiques géographiques, sociales, économiques et agricoles. Ces données sont disponibles pour la période 19962008 mais malheureusement, avec de nombreuses données manquantes ${ }^{13}$. Le RICA est une base de données européenne rassemblant des données comptables d'exploitations agricoles professionnelles. Les régions RICA correspondent aux régions retenues sauf au Royaume-Uni où l'Angleterre est divisée en seulement trois régions RICA. Les données individuelles des exploitations ont été agrégées en fonction de leur poids dans la région (défini dans la base RICA) et de leur production de lait. Nous disposons des données 1996-2006.

Les données utilisées pour les indicateurs et leur effet potentiel sur la production laitière sont résumés dans le tableau 1. Les statistiques descriptives par pays sont présentées dans l'annexe 1.

\section{La méthode}

L'analyse est scindée en deux parties. La première correspond à l'analyse de la période "actuelle". Elle fait référence aux observations moyennes sur la période 20052008 de façon à avoir au moins deux années présentes pour chaque variable. La seconde traite de l'évolution passée de la production. Elle correspond à la période 1996-2008. Dans ce cas, nous prenons pour la variable expliquée le taux d'accroissement annuel tendanciel de la densité de production laitière. Il est estimé à l'aide d'une régression linéaire de la densité de production laitière sur les années. L'estimation de ce taux à par-

13. http://epp.eurostat.ec.europa.eu/portal/page/ portal/statistics/search_database tir de la définition de points initiaux et finaux auraient d'une part nécessité que ces valeurs soient renseignées pour toutes les régions ces années là et d'autre part qu'elles ne soient pas exceptionnellement élevées ou basses. Les variables explicatives correspondent à la moyenne observée sur 1996-2008 ainsi qu'à leur taux d'accroissement sur cette même période.

Nous avons opté pour une analyse statistique simple -la corrélation- pour analyser les relations entre production laitière et caractéristiques régionales. Plusieurs raisons motivent ce choix : la taille de l'échantillon est restreinte, les données manquantes sont nombreuses et les corrélations entre les variables explicatives sont très fortes ce qui crée des problèmes de multi-colinéarité. Deux types de corrélations sont testés. Le coefficient de Pearson, le plus classiquement utilisé, suppose une relation linéaire entre deux variables et mesure la qualité de cette relation (covariance des deux variables divisée par le produit de leurs écart-types). Au lieu de prendre la valeur absolue des observations, le coefficient de Spearman estime une corrélation sur la base des rangs des observations. Cela permet d'éviter les biais liés aux valeurs extrêmes et aux non linéarités des relations. Il donne néanmoins autant de poids à un faible qu'à un fort écart entre deux observations de rangs consécutifs. Nous supposons que la mobilisation conjointe de ces deux indicateurs permettent de pallier à leurs limites respectives. Il faut tout de même rappeler que seule l'interprétation permet de différencier la cause de la conséquence entre deux variables corrélées et qu'il arrive que la dépendance apparente entre deux variables soit due en réalité aux variations d'une troisième (Saporta, 2006) $)^{14}$.

Dans chaque partie, la répartition spatiale de la production laitière et de son évolution

14. Saporta G. (2006). Probabilités, analyse des données et statistique. Ed. Technip, France. 
FAITS ET CHIFFRES

sont tout d'abord décrites. Pour interpréter les cartes, l'indicateur de Gini, proposé par Krugman $(1991)^{15}$, permet de comparer la concentration géographique de la production entre les pays. Elle varie de 0 lorsque la production est répartie uniformément entre toutes les entités à 1 si toute la production est réalisée dans une seule région. L'auto-corrélation spatiale permet d'identifier si la production est distribuée ou non de manière aléatoire entre les régions. L'indicateur de Moran (Le Gallo, 2002) $)^{16}$ s'interprète comme un coefficient de corrélation linéaire. Pour estimer cet indice, il faut en préalable considérer la position relative des unités dans l'espace. La distance à vol d'oiseau entre les centroïdes des régions est utilisée ici. Nous supposons que plus la distance est grande, moins une région a de l'influence sur une autre (Anselin, 1988) ${ }^{17}$. Ceci est modélisé en pondérant les observations des régions voisines par l'inverse de la distance au carré. Au-delà d'une certaine distance, cet effet est souvent supposé nul. Nous testons trois distances alternatives : $200 \mathrm{~km}$ qui assure une certaines proximité entre régions, $350 \mathrm{~km}$ ce qui garantit la connectivité entre deux régions voisines, et aucune distance limite afin de s'affranchir d'éventuel biais liés à la définition d'un seuil. Les poids sont standardisés par ligne de façon à ce que $1 /$ les régions soient comparables même si elles ont un nombre de voisin différent et $2 /$ pour chaque région, seule la distance relative d'un voisin par rapport à un autre importe (Larue et al., $2008^{18}$; Ben Arfa et al., 2009a, voir note 3 ).

15. Krugman P. (1991). Increasing Returns and Economics Geography. Journal of Political Economy, vol. $99, n^{\circ} 3$, p. 483-499.

16. Le Gallo (2002). Econométrie spatiale: 1'autocorrélation spatiale dans les modèles de régression linéaire. La Documentation française, Économie et prévision, $\mathrm{n}^{\circ} 155$, p. 139-157.

17 Anselin L. (1988). Spatial Econometrics : Methods and Models. Kluwer Academic, Dordrecht. 18. Larue S., Abildtrup J., Schmitt B. (2008). Modelling the Spatial structure of pig production in Denmark. 12 ${ }^{\text {th }}$ Congress of the European Association of Agricultural Economists, Ghent, Belgium.

\section{Résultats}

\section{Production laitière actuelle (moyenne 2005-2008)}

Répartition géographique de la production actuelle

Figure 1. Densité de la production laitière : moyenne 2005- $2008\left(\mathrm{t} / \mathrm{km}^{2}\right)$
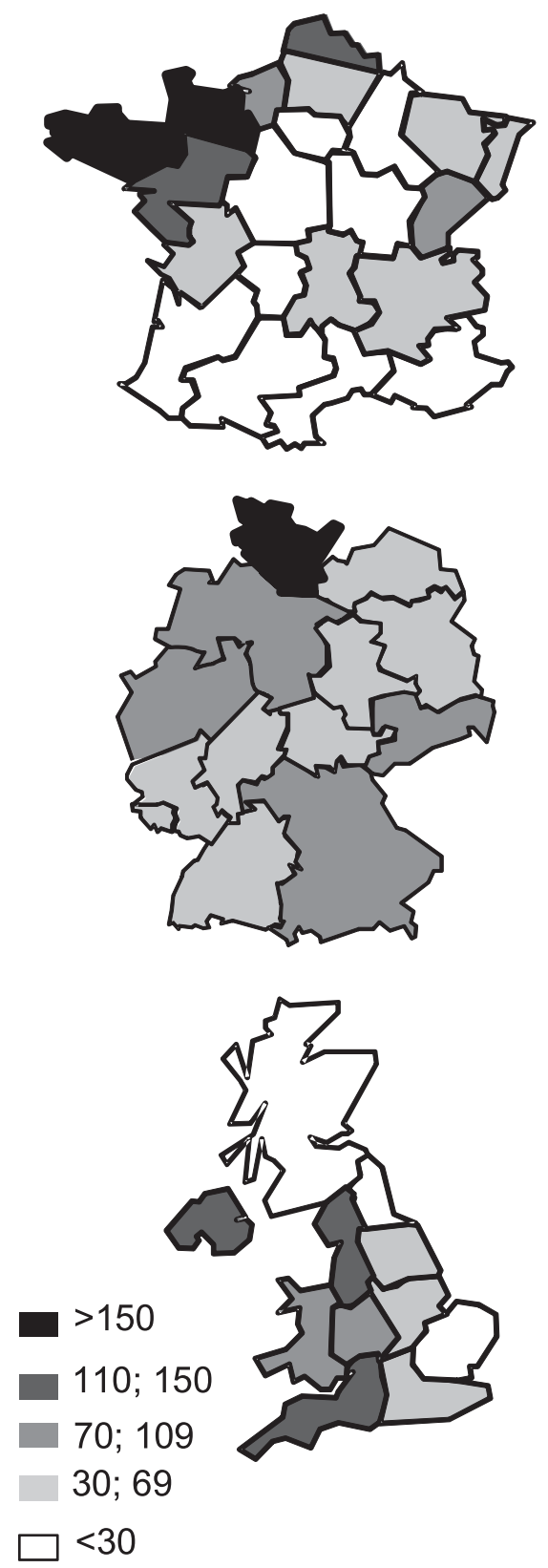
Tableau 2. Auto-Corrélation spatiale selon la distance maximale retenue entre régions voisines (moyenne 2005-2008)

\begin{tabular}{l|l|l|l}
\hline & France & Allemagne & Royaume-Uni \\
\hline $200 \mathrm{~km}$ & $0,72(p . v a l<0,00 ; \mathrm{N}=21)$ & $0,66(p . v a l=0,02 ; \mathrm{N}=12)$ & $0,38(\mathrm{p} . \mathrm{val}=0,28 ; \mathrm{N}=10)$ \\
$350 \mathrm{~km}$ & $0,88(\mathrm{p} . \mathrm{val}<0,00 ; \mathrm{N}=21)$ & $0,14(\mathrm{p} . \mathrm{val}=0,64 ; \mathrm{N}=14)$ & $0,60(\mathrm{p} . \mathrm{val}=0,04 ; \mathrm{N}=12)$ \\
Pas de limite & $0,85(\mathrm{p} . \mathrm{val}<0,00 ; \mathrm{N}=21)$ & $0,41(\mathrm{p} . \mathrm{val}=0,15 ; \mathrm{N}=14)$ & $0,47(\mathrm{p} . \mathrm{val}=0,12 ; \mathrm{N}=12)$ \\
\hline
\end{tabular}

1. La France est le pays où la production est la plus concentrée géographiquement. L'essentiel de la production est assurée par un petit nombre de région comme en atteste le coefficient de GINI qui vaut 0,509 . De plus, ces régions sont proches géographiquement.

L'auto-corrélation spatiale est la plus élevée des trois pays quelque soit la distance maximale considérée (tableau 2). Les régions du « Grand Ouest » (Bretagne, Normandie, Pays de Loire) présentent les densités de production les plus fortes tandis que le centre et le sud de la France produisent moins de $25 \mathrm{t}$ de lait par $\mathrm{km}^{2}$ (figure 1). La Bretagne est la région qui atteint la concentration la plus forte des trois pays étudiés $\left(188 \mathrm{t} / \mathrm{km}^{2}\right.$ en moyenne sur la période 2005-2008).

2. L'Allemagne a, au contraire, la répartition de la production la plus homogène (Gini : 0,250 ; faible auto- corrélation spatiale sauf lorsque l'on considère seulement des voisins très proches) : la production est relativement importante dans toutes les régions.

Les régions du Nord (Schleswig-Holstein : $155 \mathrm{t} / \mathrm{km}^{2}$; Niedersachsen : $\left.110 \mathrm{t} / \mathrm{km}^{2}\right)$ et la Bavière $\left(108 \mathrm{t} / \mathrm{km}^{2}\right)$ produisent cependant davantage et la Sarre moins avec 34 t de lait par $\mathrm{km}^{2}$.

3. La concentration de la production au Royaume-Uni est intermédiaire (Gini : $0,420)$. On observe un gradient entre l'Écosse et l'Est de l'Angleterre peu productifs $\left(<20 \mathrm{t} / \mathrm{km}^{2}\right)$, et l'Ouest du pays (jusqu'à $146 \mathrm{t} / \mathrm{km}^{2}$ en Irlande du Nord et au NordOuest de l'Angleterre).
Relation entre la densité de production laitière actuelle et les indicateurs régionaux (moyenne 2005-2008)

Globalement, on remarque que les indicateurs expliquent mieux la répartition actuelle de densité de production laitière en France (six ou sept indicateurs significatifs selon les coefficients utilisés) qu'au Royaume-Uni et en Allemagne (un à trois indicateurs significatifs) (tableau 3).

1. En France, les régions les plus productrices ont des fermes qui présentent de meilleurs résultats économiques, surtout en terme de marge nette. Le coût de production est moins fortement corrélé d'après le coefficient de Spearman que d'après celui de Pearson car bien que les régions avec de fortes densité de production comme la Bretagne aient des coûts de production globalement plus faibles $(<70 € / t)$, le nuage de point central est assez diffus. Les mouvements interrégionaux ont pourtant été très contrôlés depuis la mise en place des quotas. La répartition régionale selon les coûts de production et la marge nette s'est donc $a$ priori réalisée avant la mise en place des quotas à moins que l'agglomération de la production ait permis aux éleveurs de baisser progressivement leurs coûts de production. Ces fermes sont aussi caractérisées par des chargements plus élevés et des structures de production plus grandes (environ 300000 1/ferme en Bretagne contre 200000 1/ferme en Rhône-Alpes et Auvergne). Concernant le prix moyen du lait vendu, il augmente lorsque la densité de production s'intensifie. La différence entre les deux coefficients de corrélation s'explique par la présence de régions produc- 
FAITS ET CHIFFRES

Tableau 3. Corrélations entre la densité de production laitière actuelle et les indicateurs pris séparément (coefficients de Pearson et de Spearman) (moyenne 2005-2008)

\begin{tabular}{ll|cc|cc|cc}
\hline & & \multicolumn{2}{|c|}{ France } & \multicolumn{2}{c|}{ Allemagne } & \multicolumn{2}{c}{ Royaume-Uni } \\
\cline { 3 - 8 } & & Pearson & Spearman & Pearson & Spearman & Pearson & Spearman \\
\hline Exploitation & Marge lait/ coûts totaux & $0,51^{* *}$ & $0,66^{* * *}$ & 0,33 & 0,18 & 0,36 & 0,36 \\
& Coûts spécifiques lait & $-0,60^{* * *}$ & $-0,12$ & $-0,35$ & $-0,30$ & $-0,35$ & $-0,36$ \\
& Prix du lait & 0,15 & $0,66^{* * *}$ & $-0,35$ & $-0,29$ & $-0,55^{*}$ & $-0,35$ \\
& Rendement laitier & $-0,07$ & 0,28 & 0,10 & 0,00 & $-0,43$ & $-0,49$ \\
& Chargement & $0,41^{*}$ & $0,57^{* * *}$ & $0,56^{* *}$ & $0,78^{* * *}$ & $0,51^{*}$ & 0,44 \\
& Production lait/ ferme & $0,39^{*}$ & 0,30 & $-0,25$ & $-0,13$ & 0,30 & $0,55^{*}$ \\
\hline Agriculture & Rendement des céréales & 0,27 & $0,37^{*}$ & $0,50^{*}$ & 0,19 & n.d. & $n . d$. \\
\hline régionale & Zone défavorisée & $-0,43^{* *}$ & $-0,34$ & $-0,30$ & $-0,40$ & 0,18 & 0,26 \\
& Part des surfaces agricoles & $0,52^{* * *}$ & $0,57^{* *}$ & $0,65^{* * *}$ & $0,65 * *$ & 0,20 & 0,23 \\
& Part des exploitants $<35$ ans & $0,36^{*}$ & $0,58^{* * *}$ & 0,18 & 0,25 & 0,31 & $-0,09$ \\
& Taux d'emploi en agriculture & 0,18 & 0,01 & 0,32 & 0,30 & 0,41 & 0,32 \\
\hline Caractéristiques & Taux de chômage & $-0,19$ & $-0,21$ & $-0,23$ & $-0,04$ & $-0,54^{*}$ & $-0,33$ \\
socio-économi- & PIB /hab. & $-0,25$ & $-0,24$ & $-0,05$ & $-0,13$ & $-0,35$ & $-0,36$ \\
ques régionales & Densité de population & $-0,11$ & 0,22 & $-0,02$ & $-0,03$ & 0,07 & $-0,03$ \\
\hline
\end{tabular}

Observations : FR : $\mathrm{n}=21$, R.-U. : $\mathrm{n}=11$, ALL. : $\mathrm{n}=13$. ; n.d. : données non disponibles

${ }^{*},{ }^{* *},{ }^{* *}$ significatif à, respectivement, $10 \%, 5 \%$, et $1 \%$

trices de fromages - Rhône-Alpes, Auvergne, Franche-Comté, Nord-Pas-deCalais et Normandie - qui brouillent la relation en obtenant des prix moyen élevés (supérieurs à $300 € / t$ ) alors que la densité de production laitière est variable.

\section{Au Royaume-Uni, la production laitière} est plus dense là où le prix moyen du lait est plus faible. Ceci peut soit signifier que lorsque la production régionale est importante, il y a plus de concurrence entre éleveurs (s'ils ne sont pas organisés) et donc des prix plus bas et/ou la production laitière s'est développée dans des régions où le prix du lait est plus faible. Les unités laitières y sont plutôt de grande taille.

Dans les trois pays, les chargements élevés dans les exploitations sont associés à de forte densité de production régionale. Ceci confirmerait que les régions avec des systèmes de production plus intensifs produisent davantage. Cependant, les rendements laitiers eux ne semblent pas liés à la répartition géographique de la production actuelle. En effet, comme Chatellier et al. $(2008)^{19}$ l'ont mis en évidence, il existe une grande diversité de modèles laitiers en fonction des facteurs de production localement disponibles. Des rendements laitiers plus faibles ne veulent pas nécessairement dire que la production est moins efficace au vu des intrants utilisés. En Allemagne, la taille de l'exploitation a une dimension historique : elle est très différente entre l'ex-Allemagne de l'Est (1 500000 1/ferme) qui était organisée en grandes fermes d'État et l'ex-Allemagne de l'Ouest (180 000 1).

En Allemagne et en France, la production est plutôt localisée dans les régions caractérisées par une bonne qualité des terres agricoles, ce qui est contraire à notre l'hypothèse : les régions qui ont des rendements en céréales élevés ont un élevage laitier plus développé et la production est moins importante dans les zones classée « défavorisée » (ce classement européen prend en compte les potentialités agricoles et des aspects socio-économiques régionaux). Pour les trois pays (mais les corré-

19. Chatelier V., Pflimlin A. Perrot C. (2008). La production laitière dans les régions de l'arc Atlantique européen. Inra, Production animale, vol. 21, $\mathrm{n}^{\circ} 5$, p. $427-440$. 
Tableau 4. Auto-Corrélation spatiale entre les évolutaions de production laitière régionale selon la distance maximale retenue entre régions voisines (tendance 1996-2008)

\begin{tabular}{|c|c|c|c|}
\hline & France & Allemagne & Royaume-Uni \\
\hline $200 \mathrm{~km}$ & $0,51$ (p.val $<0,02 ; N=21)$ & 0,77 (p.val = 0,02; N=9) & 0,04 (p.val = 0,92; N=8) \\
\hline $350 \mathrm{~km}$ & $0,51$ (p.val $<0,02 ; N=21)$ & 0,74 (p.val $=0,00 ; N=13$ ) & $0,09$ (p.val $=0,80 ; N=10)$ \\
\hline Pas de limite & $0,42$ (p.val $<0,06 ; N=21)$ & $0,75$ (p.val $=0,00 ; N=13)$ & $0,45$ (p.val $=0,16 ; N=11)$ \\
\hline
\end{tabular}

lations ne sont pas toujours significatives), les régions rurales avec une part importante de terres agricoles, des agriculteurs jeunes et un fort taux d'emploi agricole sont favorables à la production laitière. L'élevage laitier semble profiter d'un contexte régional favorable à l'agriculture.

Peu des indicateurs socio-économiques étudiés apparaissent significatifs dans l'explication de la répartition de la production laitière actuelle. Au Royaume-Uni, la production laitière apparaît cependant plus faible dans les régions où le taux de chômage est bas.

La production laitière, conclusion. La France a
la production la plus concentrée des trois pays
malgré une politique de quota qui a limité
les mouvements interrégionaux de produc-
tion laitière à partir de 1984. L'Allemagne a la
répartition de la production la plus homo-
gène sur son territoire. Étonnamment, ce n'est
pas au Royaume-Uni mais en France que la
production actuelle est la plus fortement liée
aux bons résultats économiques des exploita-
tions. Par ailleurs, il semble que la production
laitière soit présente surtout dans les régions
où le secteur agricole est important, le poten-
tiel agronomique relativement bon et les char-
gements animaux élevés.

\section{2. Évolution de la production laitière}

L'accroissement des densités de production laitières dans les différentes régions (période 1996-2008)

1. En France, un léger déclin de la densité de production laitière apparaît dans les régions du sud et de l'est de la France, et de façon plus prononcée en Alsace $\left(-0,5 \mathrm{t} / \mathrm{km}^{2} /\right.$ an soit environ $13 \%$ de perte entre 1995 et 2008) (figure 3). En revanche, aucune région n'enregistre de tendance à l'augmentation clairement positive, ce qui est logique compte tenu du mode de gestion des quotas.

2. En Allemagne, les évolutions sont plus contrastées entre des régions du sud-ouest du pays en perte de vitesse et des régions en progression telles que le SchleswigHolstein dans le nord $\left(+0,5 \mathrm{t} / \mathrm{km}^{2} / \mathrm{an}\right)$ et $\mathrm{l}^{\prime}$ ex-RDA (jusqu'à $+0,7$ t/ $\mathrm{km}^{2} / \mathrm{an}$ soit environ $+13 \%$ de 1995 à 2008). La réserve nationale avait en effet tendance à allouer les quotas disponibles aux régions qui avaient les plus fortes demandes (Alliance Environnement, 2008) ce qui a donné un peu de souplesse aux échanges.

3. Au Royaume-Uni, les dynamiques sont encore plus tranchées. On observe un transfert de la production du sud-est vers le nord-ouest. Les régions anglaises enregistrent des chutes de production très importantes, et ce particulièrement dans le sud de l'Angleterre $\left(-1,8 \mathrm{t} / \mathrm{km}^{2} /\right.$ an soit environ $-10 \%$ sur la période 1995-2008), alors que l'Irlande du Nord intensifie sa production laitière à un rythme bien supérieur à ceux rencontrés dans les autres régions étudiées $\left(+3,2 \mathrm{t} / \mathrm{km}^{2} / \mathrm{an}\right.$ ou $+25 \%$ sur la même période). La comparaison des écarts types (test de Levenne) confirme que les transferts inter-régionaux de production ont été significativement plus faibles en France (écart type : $0,15 \mathrm{t} / \mathrm{km}^{2} / \mathrm{an}$ ) qu'au Royaume-Uni (1,31 t/km²/an). De même, alors que la production s'est davantage concentrée dans les trois pays étudiés, les processus de concentration ont été plus importants au Royaume-Uni (GINI : 
Figure 3. Taux d'évolution tendanciel de la production laitière $\left(\mathrm{t} / \mathrm{km}^{2} / \mathrm{an}\right)$
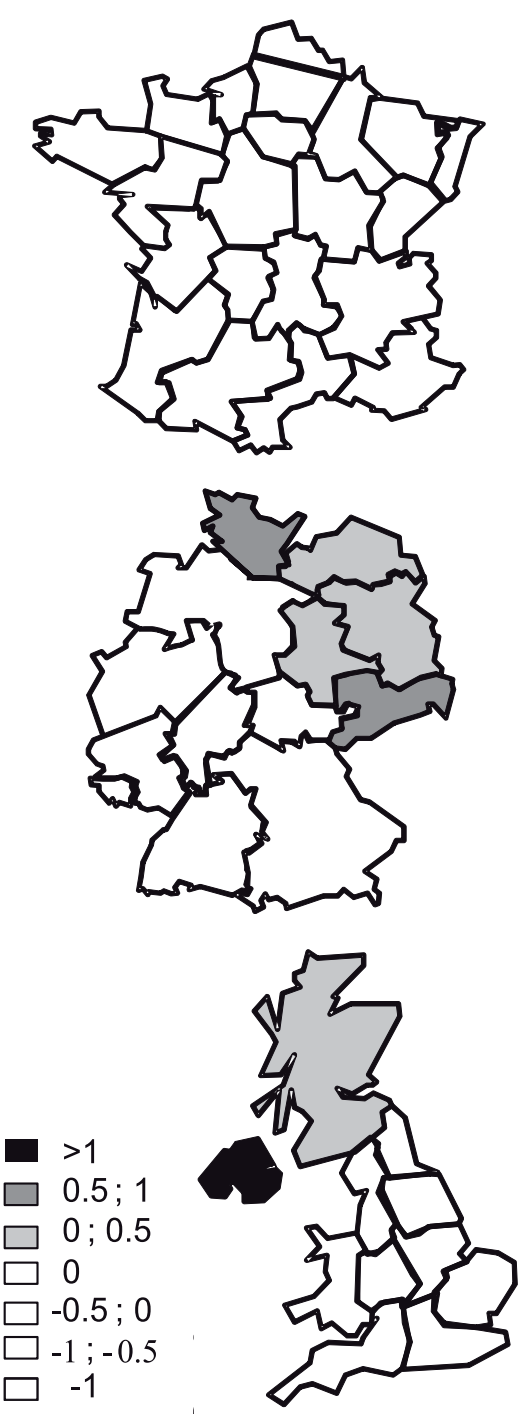

$+0,043$ entre 2001 et $\left.2008^{20}\right)$ qu'en France $(+0,006)$ et en Allemagne $(+0,012)$.

L'auto-corrélation spatiale de ces dynamiques est positive, elle est particulièrement significative en Allemagne (tableau 4). Cela laisse supposer qu'il y a un effet d'entraînement entre les régions proches géographiquement.

20. 2001 est l'année la plus ancienne pour laquelle il n'existe aucune donnée manquante.
Relation entre l'accroissement de la densité de production laitière et les indicateurs moyens régionaux (période 1996-2008)

Le tableau 5 montre que beaucoup d'indicateurs moyens sur la période 1996-2008 sont très significativement corrélés à l'accroissement de la production laitière au Royaume-Uni (neuf significatifs), assez significativement corrélés en Allemagne (huit). Seul un indicateur est significativement corrélé aux changements de densité de production régionale en France. Cela fait écho à l'intensité des modifications de densité production laitière observée dans ces pays.

1. Au Royaume-Uni, en moyenne sur la période étudiée, de faibles coûts de production et des prix du lait bas, une marge nette importante, un faible potentiel agricole mais un secteur agricole dominant sont les caractéristiques des zones de fort accroissement de production laitière (tableau 3 ). Ceci est en accord avec notre hypothèse de départ : les mouvements de production ont été plus importants au Royaume-Uni, et ceux-ci ont été en faveur des régions qui présentaient des avantages comparatifs : une bonne compétitivité de la production laitière, un secteur agricole prédominant et un potentiel agronomique limité. La faible densité de la population apparait aussi comme un élément favorable : dans les zones moins peuplées, le coût de la terre est souvent plus bas et les nuisances liées à la production sont moins problématiques. La production s'est développée dans les régions dont les rendements laitiers étaient plus faibles (6 $800 \mathrm{~L} / \mathrm{vache}$ en Irlande du Nord vs 7500 L/vache en Angleterre). Cependant ils s'améliorent : en Irlande du Nord, ces rendements sont passés de 5440 L/Vache en 1996 à 6790 L/vache en 2006.

2. En Allemagne, la production laitière augmente dans les régions présentant de grandes exploitations avec des rendements laitiers importants et des prix du lait bas. 
Tableau 5. Corrélations entre le taux d'accroissement tendanciel de la densité de production laitière entre 1996 et 2008 et les indicateurs moyens ${ }^{1}$ pris séparément (coefficients de Pearson et de Spearman) (moyenne 2005-2008)

\begin{tabular}{|c|c|c|c|c|c|c|c|}
\hline & \multicolumn{2}{|c|}{ France } & \multicolumn{2}{|c|}{ Allemagne } & \multicolumn{2}{|c|}{ Royaume-Uni } \\
\hline & & Pearson & Spearman & Pearson & Spearman & Pearson & Spearman \\
\hline & $\begin{array}{l}\text { Densité moyenne de } \\
\text { production laitière }\end{array}$ & $-0,19$ & $-0,10$ & 0,50 * & 0,41 & 0,43 & 0,1 \\
\hline Exploitation & $\begin{array}{l}\text { Marge nette du lait } \\
\text { Coûts spécifiques du lait } \\
\text { Prix du lait } \\
\text { Rendement laitier } \\
\text { Chargement herbivore } \\
\text { Production de lait par ferme }\end{array}$ & $\begin{array}{r}0,36 \\
-0,36 \\
-0,24 \\
-0,18 \\
-0,25 \\
0,15\end{array}$ & $\begin{array}{r}0,15 \\
-0,09 \\
0,11 \\
-0,33 \\
-0,22 \\
0,00\end{array}$ & $\begin{array}{c}-0,37 \\
0,32 \\
-0,50^{*} \\
0,58^{* *} \\
-0,05 \\
0,46^{*}\end{array}$ & $\begin{array}{l}-0,34 \\
0,32 \\
-0,71 * * * \\
0,51^{*} \\
0,03 \\
0,73 * * *\end{array}$ & $\begin{array}{c}0,84^{* * *} \\
-0,85^{* * *} \\
-0,57^{* *} \\
-0,84^{* * *} \\
0,28 \\
0,25\end{array}$ & $\begin{array}{l}0,88^{* * *} \\
-0,88^{* * *} \\
-0,64 * * \\
-0,64 * * \\
-0,11 \\
-0,30\end{array}$ \\
\hline $\begin{array}{l}\text { Agriculture } \\
\text { régionale }\end{array}$ & $\begin{array}{l}\text { Rendement des céréales } \\
\text { Zone défavorisée } \\
\text { Part des surfaces agricoles } \\
\text { Part des exploitants }<35 \text { ans } \\
\text { Taux d'emploi en agriculture }\end{array}$ & $\begin{array}{c}-0,46^{* *} \\
0,12 \\
0,03 \\
0,21 \\
0,16\end{array}$ & $\begin{array}{l}-0,23 \\
-0,09 \\
0,19 \\
0,65^{* *} \\
0,17\end{array}$ & $\begin{array}{c}0,18 \\
-0,35 \\
0,60 * \star \\
0,04 \\
0,48 *\end{array}$ & $\begin{array}{c}0,13 \\
-0,44 \\
0,79 * * * \\
-0,04 \\
0,64 * *\end{array}$ & $\begin{array}{l}-0,62^{* *} \\
0,69 * * * \\
0,40 \\
0,90^{* * *} \\
0,81 * * *\end{array}$ & $\begin{array}{l}-0,66 * * \\
0,87 * * * \\
0,02 \\
0,89 * * * \\
0,30\end{array}$ \\
\hline $\begin{array}{l}\text { Caractéristique } \\
\text { socio-économ } \\
\text { ques régionale }\end{array}$ & $\begin{array}{l}\text { Taux de chômage } \\
\text { PIB/hab. } \\
\text { Densité de population }\end{array}$ & $\begin{array}{l}0,17 \\
0,17 \\
0,08\end{array}$ & $\begin{array}{r}0,00 \\
0,18 \\
-0,09\end{array}$ & $\begin{array}{l}0,52^{*} \\
-0,58^{* *} \\
-0,30\end{array}$ & $\begin{array}{l}0,79 * * * \\
-0,57^{*} \\
-0,52^{*}\end{array}$ & $\begin{array}{l}-0,31 \\
-0,45 \\
-0,50^{*}\end{array}$ & $\begin{array}{r}0,53^{*} \\
-0,55^{*} \\
-0,53\end{array}$ \\
\hline
\end{tabular}

Observations: $\mathrm{FR}: \mathrm{n}=21, \mathrm{RU}: \mathrm{n}=11, \mathrm{ALL}: \mathrm{n}=13 .{ }^{*},{ }^{* *}{ }^{*}{ }^{* * *}$ respectivement significatif à $10 \%, 5 \%$ et $1 \%$

1. moyenne 1996-2008

Ces régions sont des régions rurales et agricoles avec peu d'alternatives : le taux de chômage y est plus élevé, le PIB par habitant et la densité de population relativement faibles. Ces corrélations sont tirées par les länder de l'ex-Allemagne de l'Est qui ont une situation économique plus difficile et qui bénéficie d'une croissance de la production importante.

3. En France, seul le rendement des céréales ou la part de jeunes exploitants apparaissent significatifs. Ceci pourrait s'expliquer par la régression de la production laitière dans l'est de la France qui a des terres arables avec de bons potentiels agronomiques et un secteur " grandes cultures » plus dynamique. En France, étant donné que les mouvements interrégionaux sont contrôlés, la production ne diminue pas parce qu'il est plus avantageux de produire du lait ailleurs mais parce que localement d'autres activités sont plus rentables.

Dans l'ensemble (même signe pour les trois pays mais pas forcément significatif), la production a tendance à augmenter plutôt dans les régions caractérisées par une part importante de surface agricole, un taux d'emploi en agriculture élevé, une population d'agriculteurs jeune et dans lesquelles la production de lait par exploitation est importante.

\section{Relation entre l'accroissement de la densité} de production laitière avec l'accroissement de certains indicateurs régionaux (période 1996-2008)

L'évolution des volumes de lait produit par région s'est accompagnée de changements des caractéristiques des exploitations, du secteur agricole et des caractéristiques socioéconomiques de la région. Les relations significatives entre ces évolutions parallèles sont plus nombreuses pour le RoyaumeUni (tableau 6).

1. Au Royaume-Uni, l'accroissement de la production laitière s'est accompagné d'une baisse des coûts de production. Le développement du secteur laitier peut également favoriser l'implantation de service et d'industries qui vont générer des économies externes pour les exploitations et qui permettent in fine de réduire les coûts 
FAITS ET CHIFFRES

Tableau 6. Corrélations entre le taux d'accroissement tendanciel de la densité de production laitière entre 1996 et 2008 et le taux d'accroissement des indicateurs pris séparément (coefficients de Pearson et de Spearman)

\begin{tabular}{ll|cc|cc|cc}
\hline & \multicolumn{2}{|c|}{ France } & \multicolumn{2}{c|}{ Allemagne } & \multicolumn{2}{c}{ Royaume-Uni } \\
\cline { 3 - 8 } & & Pearson & Spearman & Pearson & Spearman & Pearson & Spearman \\
\hline Exploitation & Marge nette du lait/ coûts tot. & 0,04 & $-0,13$ & $0,50^{*}$ & 0,28 & 0,37 & $0,64^{* *}$ \\
& Coûts spécifique du lait & $-0,14$ & $-0,28$ & $-0,18$ & 0,13 & $-0,54^{*}$ & $-0,78^{* * *}$ \\
& Prix du lait & 0,10 & 0,00 & 0,09 & 0,02 & $-0,58^{*}$ & $-0,26$ \\
& Rendement laitier & $0,54^{* *}$ & 0,28 & $0,60^{* *}$ & $0,74^{* * *}$ & 0,30 & 0,09 \\
& Chargement herbivore & $0,47^{* *}$ & 0,28 & 0,28 & 0,43 & 0,40 & 0,40 \\
& Production de lait par ferme & 0,26 & $0,52^{* *}$ & 0,43 & $0,73^{* * *}$ & 0,43 & $0,65^{* *}$ \\
\hline Agriculture & Rendement des céréales & $-0,14$ & 0,20 & $-0,03$ & $-0,11$ & n.d. & n.d. \\
régionale & Part des surfaces agricoles & 0,05 & 0,10 & $-0,10$ & 0,05 & $-0,64^{* *}$ & $-0,51$ \\
& Part des exploitants <35 ans & 0,25 & $-0,01$ & 0,25 & $-0,33$ & $-0,54^{*}$ & $-0,57^{*}$ \\
& Taux d'emploi en agriculture & 0,10 & 0,26 & $-0,06$ & $-0,33$ & $-0,71^{* *}$ & $-0,19$ \\
\hline Caractéristiques Taux de chômage & $-0,39 *$ & $-0,29$ & $-0,19$ & $-0,30$ & $-0,56^{*}$ & $-0,55^{*}$ \\
socio-économi- PIB/hab. & $0,44^{* *}$ & $0,49^{* *}$ & $-0,41$ & $-0,46$ & $-0,17$ & $-0,26$ \\
ques régionales & Densité de population & $-0,05$ & 0,21 & $-0,42$ & $-0,36$ & 0,29 & $0,65^{* *}$ \\
\hline
\end{tabular}

Observations: $F R: n=21, R U: n=11, A L L: n=13 .{ }^{*},{ }^{* *}{ }^{*}{ }^{* * *}$ significatif respectivement à $10 \%, 5 \%$ et $1 \%$ n.d : nombre insuffisant de données disponibles pour estimer la tendance

Evolution de la production laitière, conclusion. Comme on s'y attendait, les conséquences des choix politiques en matière de transfert des quotas laitiers sont visibles sur les évolutions passées : plus le système était libéral, plus les changements ont été importants, aboutissant à une augmentation de la concentration géographique de la production laitière dans des régions spatialement proches.

Au Royaume-Uni, les régions bénéficiant d'un accroissement de la production laitière sont celles dont les exploitations présentent la baisse des coûts de production la plus forte. Des prix du lait bas et en diminution caractérisent ces régions, traduisent un environnement très concurrentiel. En Allemagne, les régions qui voient leur production augmenter sont surtout les régions d'Allemagne de l'est caractérisées par un secteur économique plutôt à la traîne. C'est plutôt la productivité laitière des vaches qui s'améliorent que les résultats économiques. En France, la diminution de production laitière des régions se traduirait par une extensification de la production au niveau des exploitations.

de production. Les prix du lait se sont aussi davantage dégradés dans ces régions, l'amélioration de la marge nette est ainsi moins nette. Ceci peut s'expliquer par une concurrence plus forte qui tire les prix et coûts de production vers le bas, surtout lorsque le pouvoir de négociation des éleveurs est faible. Dans ces régions, la part du secteur agricole dans l'emploi total a plutôt régressé mais parallèlement le taux de chômage a diminué. L'environnement économique semble s'être globalement amélioré ce qui a permis à une partie de la population agricole de changer de secteur d'activité. La baisse de la part du secteur agricole n'a pas nuit au développement de la production laitière. Il est possible cependant que ce soit d'autres productions agricoles qui aient régressé.

2. En Allemagne, on observe que l'augmentation de la production a été associée à une amélioration des rendements laitiers et dans une moindre mesure de la marge nette. Contrairement au Royaume-Uni où c'est avant tout la diminution des coûts qui est recherché, il semble qu'en Allemagne l'accent soit mis sur la recherche de gain de productivité.

3. En France, les baisses de production ont eu lieu plus particulièrement dans les régions qui ont moins intensifié la production (ren- 
dement laitier et chargement) et pour lesquelles les conditions économiques régionales se sont plutôt dégradées.

Dans l'ensemble, l'augmentation de la production laitière s'accompagne d'une amélioration des résultats économiques plus ou moins prononcée, d'une intensification des systèmes de production (augmentation des rendements laitiers et des chargements) et d'une tendance à l'agrandissement des exploitations.

\section{Conclusion}

L'originalité de cette étude réside tout d'abord dans la comparaison des dynamiques régionales au sein de trois pays de l'Union européenne aux politiques en matière de gestion des quotas très diverses : la France qui est très interventionniste et qui cherche à limiter les déplacements géographiques de la production, le RoyaumeUni qui, au contraire, n'impose quasiment aucune restriction sur les échanges de quotas et l'Allemagne qui se trouve dans une situation intermédiaire. Ensuite, elle cherche à relier les quantités de lait produites et son évolution aux variables ayant trait aux résultats techniques et économiques des exploitations - y compris à leurs coûts de production -, au secteur agricole et aux caractéristiques socio-économiques des régions afin de vérifier si la production laitière s'organise selon les avantages comparatifs de chaque région.

Les principaux résultats sont les suivants. Comme on pouvait le présager, les politiques de transferts de quotas les plus libérales ont conduit à des évolutions plus fortes des volumes de lait produit par région.

Le Royaume-Uni a ainsi enregistré les progressions (ex : Irlande du Nord) et les régressions les plus fortes (Angleterre du sud) des trois pays. À l'opposé, seules quelques régions françaises ont vu régresser un peu leur production (ex l'Alsace) tandis qu'elle restait stable partout ailleurs. En
Allemagne, ce sont les länder d'ex-Allemagne de l'est ainsi que le nord de l'Allemagne qui ont bénéficié des transferts de production. Partout, ces transferts ont contribué à accentuer la concentration géographique de la production laitière dans des régions spatialement proches. L'agglomération permet en effet de générer des économies externes en favorisant l'implantation de services dédiés, et surtout en facilitant la collecte et le traitement du lait par les entreprises aval qui ont tendance elles-aussi à se regrouper. Les politiques de quotas plus interventionnistes n'ont cependant pas pu inverser les tendances passées. Malgré les efforts de l'administration française pour éviter une concentration supplémentaire des activités de production laitière, il s'avère que des trois pays, c'est toujours celui qui enregistre la concentration la plus forte de la production, avec des densités particulièrement élevées en Bretagne.

Au Royaume-Uni, les transferts de production ont été très importants en direction des régions qui ont les plus faibles coûts de production et les prix du lait les plus bas. Pourtant, la production actuelle n'est que faiblement corrélée aux résultats économiques des exploitations et au prix du lait. Cela laisse penser que la restructuration du secteur laitier va se poursuivre. Étant donné que, dans les régions où la production se développe, les prix du lait baissent plus fortement qu'ailleurs, on peut cependant se demander dans quelle mesure les éleveurs vont pouvoir suivre. La situation est presque opposée en France. Malgré les faibles variations de production enregistrées, la production actuelle est en effet localisée dans les zones où les exploitations obtiennent les meilleurs résultats économiques et où les exploitations sont les plus grandes. La restructuration à venir ne sera alors peut être pas aussi importante qu'au Royaume-Uni. En Allemagne, les transferts de production ont surtout bénéficié aux länder de l'ex-Allemagne de l'Est qui sont caractérisés par un secteur écono- 
mique en retrait par rapport au reste de l'Allemagne et par des exploitations de très grande taille avec des rendements laitiers élevés. Ces mouvements ne semblent pas guidés par les bons résultats économiques des exploitations mais plutôt par les faibles coûts du lait et les plus faibles coûts d'opportunité. Dans ces régions, la marge nette s'améliore cependant et la densité de production laitière reste peu élevée. L'Allemagne est le pays où la production est répartie de façon la plus homogène malgré des systèmes de production très divers. $\mathrm{La}$ question est tout de même de savoir si le nord, qui a une production plus industrielle va continuer à accroître sa production qui est déjà la plus élevée d'Allemagne.

Les limites de cette étude descriptive ont principalement trait au faible nombre de données et à leur qualité. De plus, la méthode retenue ne permet de faire que des suppositions quant à l'évolution future de la répartition de la production après l'abolition des quotas. Une analyse des investissements réalisés par les éleveurs et les filières offri- rait un autre éclairage en mettant en évidence les régions qui misent sur la production laitière future. De plus, bien que cette étude confirme que les dynamiques semblent aller vers une concentration accrue de la production, elle ne dit pas jusqu'à quel niveau de concentration les processus d'agglomération permettent d'améliorer les performances économiques des exploitations et de la filière, et à partir de quand les problèmes environnementaux deviennent vraiment limitant. De plus, les processus de réorganisation spatiale et de concentration de la production ne sont pas garant d'une meilleure compétitivité au niveau national. En effet, le Royaume-Uni qui a eu la réorganisation spatiale la plus forte est le pays qui a vu sa production nationale le plus chuter (Commission européenne, 2007; IPTS, 2009) ${ }^{21}$.

\section{Remerciements}

Les auteurs remercient les remarques et suggestions des relecteurs extérieurs anonymes qui ont permis d'améliorer cet article.

21. Commission européenne (2007). Milk margins in the European Union (2004) http://ec.europa.eu/ agriculture/rica/pdf/sa0202_milk_2007.pdf

Institute for Prospective Technological Studies (IPTS) (2009). Economic Impact of the Abolition of the milk Quota Regime. Regional analysis of the Milk Production in the EU. http://ec.europa.eu/ agriculture/analysis/external/milkquota/ex_sum_en.pdf 


\begin{tabular}{|c|c|c|c|c|c|c|c|c|c|c|c|}
\hline \multirow{5}{*}{ 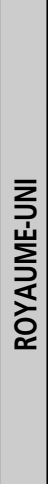 } & $\sum^{\frac{x}{2}}$ & & $\frac{6}{m}$ & 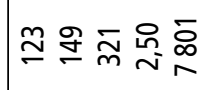 & రి & $\frac{m}{\infty} \frac{\infty}{\infty}$ & $\begin{array}{l}0^{0} \\
=\end{array}$ & $\begin{array}{l}20 \\
i\end{array}$ & in & 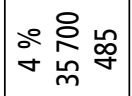 & \\
\hline & $\stackrel{\varrho}{\Sigma}$ & $\infty$ & $\stackrel{\Upsilon}{\leftarrow}$ & 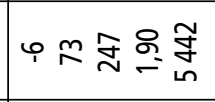 & 유 & レ & 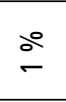 & 운 & $\stackrel{0}{-}$ & 윰 & \\
\hline & 竞 き & 요 & $\stackrel{\mathscr{Z}}{\cong}$ & 必 & ஜ & $\infty$ o̊ & $\stackrel{\circ}{\sim}$ & in & $\stackrel{2}{-}$ & ஃ̊ & \\
\hline & $\dot{\bar{\sigma}}$ & $\mathscr{\circ}$ & $\begin{array}{c}\bar{f} \\
\\
1\end{array}$ & nి ષ & $\stackrel{\infty}{\forall}$ & $8 \stackrel{\circ}{\mathrm{n}}$ & $\stackrel{+}{\circ}$ & $\begin{array}{l}\stackrel{0}{0} \\
\infty\end{array}$ & $\stackrel{\circ}{\sim}$ & 용 & \\
\hline & ڤัँ & $\cong$ & $=$ & 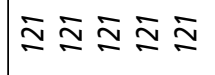 & $\cong$ & $\stackrel{\infty}{\sim} \underset{⿱}{\Sigma}$ & $\stackrel{\Re}{\Xi}$ & $\stackrel{\Re}{\Sigma}$ & $\stackrel{ }{=}$ & 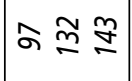 & \\
\hline \multirow{5}{*}{ 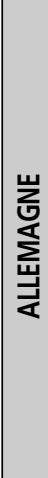 } & 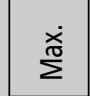 & ํㅛㅁ & $\frac{\hbar}{0}$ & 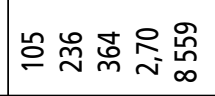 & 음 & ळ & $\begin{array}{l}\text { å } \\
\text { a }\end{array}$ & $\frac{0}{6}$ & $\stackrel{0}{1}$ & 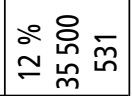 & \\
\hline & $\stackrel{. \check{E}}{\Sigma}$ & $\vec{m}$ & 辇 & 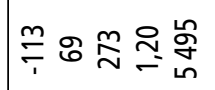 & 으 & $\stackrel{m}{m} \stackrel{0}{\circ}$ & $\stackrel{\circ}{m}$ & $\begin{array}{l}\stackrel{0}{\infty} \\
\stackrel{\sim}{\infty}\end{array}$ & $\stackrel{\circ}{-}$ & 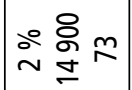 & \\
\hline & 豙 & $\stackrel{m}{m}$ & $\tilde{m}$ & 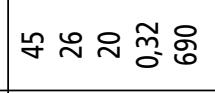 & 点 & の & $\stackrel{\circ}{m}$ & $\begin{array}{l}\circ \\
\text { 음 }\end{array}$ & $\therefore$ & 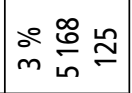 & \\
\hline & ¿ें & $\mathbb{N}$ & $\stackrel{n}{\circ}$ & 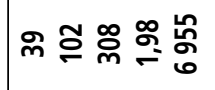 & ஜิ & ఫ̛ & $\stackrel{\circ}{\circ}$ & \& & $\stackrel{\circ}{m}$ & 용 & \\
\hline & $\stackrel{\nu}{\Sigma}$ & $\stackrel{m}{\Sigma}$ & $m$ & 언 안 원 언 & எ & 응 & $\lesssim$ & 응 & $\stackrel{\infty}{\simeq}$ & $\cong \stackrel{2}{1}$ & \\
\hline \multirow{5}{*}{ 岁 } & $\stackrel{\sqrt[x]{\pi}}{\Sigma}$ & హ) & 0 & 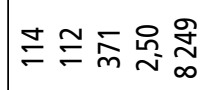 & $\stackrel{\circ}{m}$ & 응 & $\begin{array}{l}\dot{0} \\
\text { Oे }\end{array}$ & $\begin{array}{l}\circ \\
\text { ò }\end{array}$ & $\stackrel{\circ}{=}$ & 융 & \\
\hline & $\dot{\Sigma}$ & $r$ & $\begin{array}{l}\text { g } \\
0 \\
1\end{array}$ & - ஐ $\stackrel{\infty}{\sim} \stackrel{\circ}{\circ} \stackrel{9}{\circ}$ & 요 & ని & 웅 & $\begin{array}{l}\therefore 0 \\
0\end{array}$ & $\therefore$ & 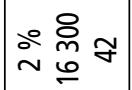 & \\
\hline & 竞 き & 유 & $\frac{\operatorname{Ln}}{\sigma}$ & 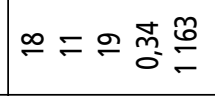 & ํำ & $\stackrel{\circ}{\stackrel{\circ}{m}}$ & $\stackrel{\circ}{m}$ & $\stackrel{\circ}{ \pm}$ & $\stackrel{\circ}{m}$ & 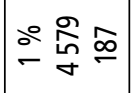 & - \\
\hline & ¿ें & in & $\begin{array}{l}8 \\
0 \\
0 \\
1\end{array}$ & స & రి & ஃ & $\stackrel{\circ}{\simeq}$ & 望 & in & 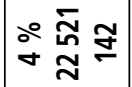 & \\
\hline & ڤั้ & & $\bar{\sim}$ & 응 응 ลે & స్ & 윯 & $\stackrel{m}{\lesssim}$ & $\stackrel{\Re}{\lesssim}$ & $\stackrel{\circ}{\sim}$ & @ స్ స & \\
\hline \multicolumn{2}{|r|}{$\begin{array}{l}\frac{0}{0} \\
\frac{.0}{\frac{0}{5}} \\
\frac{10}{7}\end{array}$} & & 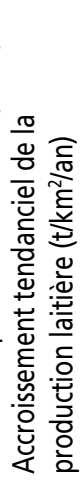 & 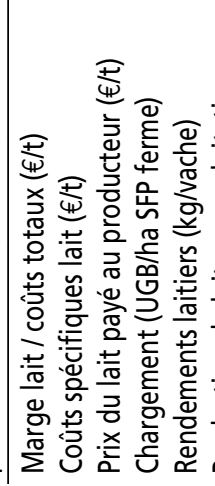 & 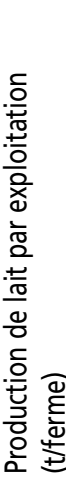 & 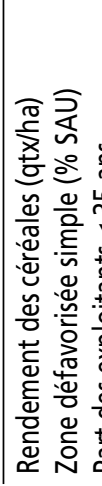 & 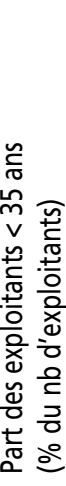 & 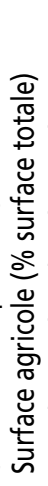 & 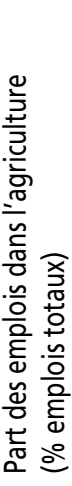 & 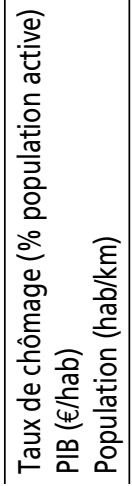 & . \\
\hline
\end{tabular}

